\title{
Does Cascading Schmitt-Trigger Stages Improve the Metastable Behavior?
}

\author{
Andreas Steininger and Robert Najvirt and Jürgen Maier \\ Vienna University of Technology, 1040 Vienna, Austria \\ \{steininger, rnajvirt, jmaier\}@ecs.tuwien.ac.at
}

\begin{abstract}
Schmitt-Trigger stages are the method of choice for robust discretization of input voltages with excessive transition times or significant noise. However, they may suffer from metastability. Based on the experience that the cascading of flip-flop stages yields a dramatic improvement of their overall metastability hardness, in this paper we elaborate on the question whether the cascading of Schmitt-Trigger stages can obtain a similar gain.

We perform a theoretic analysis that is backed up by an existing metastability model for a single Schmitt-Trigger stage and elaborate some claims about the behavior of a Schmitt-Trigger cascade. These claims suggest that the occurrence of metastability is indeed reduced from the first stage to the second which suggests an improvement. On the downside, however, it becomes clear that metastability can still not be completely ruled out, and in some cases the behavior of the cascade may be less beneficial for a given application, e.g. by introducing seemingly acausal transitions. We validate our findings by extensive HSPICE simulations in which we directly cover our most important claims.
\end{abstract}

\section{INTRODUCTION}

In VLSI design two types of discretization are performed to make real-world quantities "computable" in a digital domain. The values of originally analog quantities are transformed from their continuous space into a suitable digital (i.e. discrete) representation by analog-to-digital converters. In the time domain a periodic clock signal performs a transformation from a continuous to a discrete time by sampling these digital logic states at suitable instances, typically the clock edge. It has been formally proven that every such transformation from a continuous space to a discrete one inevitably introduces the risk of metastability [1], i.e. a prolonged state of indecision between two discrete values. Consequently one either has to allow an unbounded decision time, or expect to occasionally experience undefined, also known as "metastable", readings. In the time domain this question boils down to deciding about the precedence of events; it has been extensively studied in context with flip-flops [2], where a decision needs to be made whether a transition on a data line occurred before or after a given clock edge. Synchronizer circuits are used to reduce the risk of metastable upsets to a suitable level, albeit at the cost of performance. In case of the waiting synchronizer [3] flipflop stages are simply cascaded to that end, which generally yields a dramatic improvement in reliability.

This research was partially supported by the SIC project (grant P26436-N30) of the Austrian Science Fund (FWF).

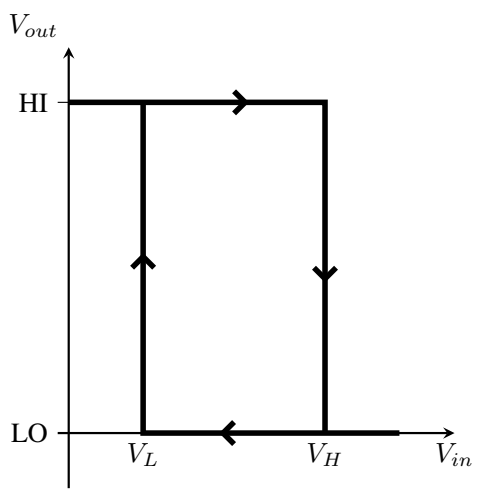

Fig. 1. S/T hysteresis

In the value domain the most fundamental task is that of a discriminator which has to decide whether a given input voltage is higher or lower than a reference. To avoid oscillating behavior for inputs close to the reference, SchmittTrigger (S/T) stages [4] are employed instead whose hysteresis behavior makes them ignore irrelevant voltage fluctuations. However, it has been shown that for certain input traces a $\mathrm{S} / \mathrm{T}$ can become metastable as well [5], [6]. Following the lessons learned from synchronizers, one may ask whether the cascading of $\mathrm{S} / \mathrm{Ts}$ is again effective in reducing the risk of metastable upsets. This is exactly the question we want to address in this paper.

To this end we will, in the next section, revisit the behavior of a single $\mathrm{S} / \mathrm{T}$ stage. Based on this knowledge we will, in Section III, break down our key question into sub-questions whose answer may finally allow the desired overall judgment. In Section IV we will briefly introduce the metastability model for a single S/T stage, as derived by Marino in [5] and use it to shed light on our related sub-questions. On the foundation of this analysis we will elaborate predictions for the behavior of a two-stage $\mathrm{S} / \mathrm{T}$ cascade in Section V which we will validate by extensive simulation experiments in Section VI. Finally, we will conclude the paper with Section VII.

\section{Behavior of A Single Schmitt-Trigger Stage}

The key property of a $\mathrm{S} / \mathrm{T}$ is its hysteresis behavior as shown in Fig. 1. Once an input voltage $V_{i n}$ has crossed the upper threshold $V_{H}$ from below, the $\mathrm{S} / \mathrm{T}$ output flips

(C) 2016 IEEE. Personal use of this material is permitted. Permission from IEEEmust be obtained for all other uses, in any current or future media, includingreprinting/republishing this material for advertising or promotional purposes,creating new collective works, for resale or redistribution to servers or lists,or reuse of any copyrighted component of this work in other works. 


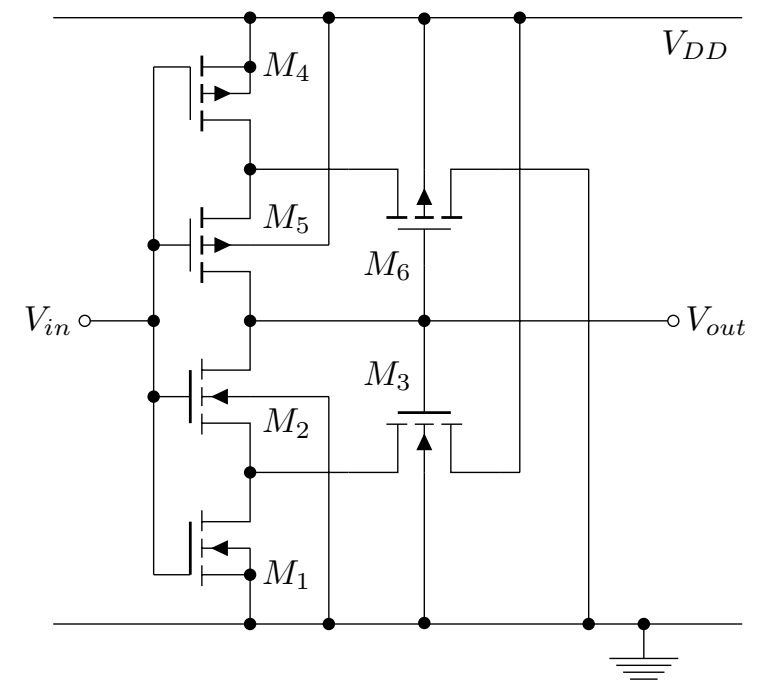

Fig. 2. Conventional CMOS S/T implementation (from [4])

to $\mathrm{LO}^{1}$ and will not return to $\mathrm{HI}$ before the input crosses the lower threshold $V_{L}$. This behavior provides the $\mathrm{S} / \mathrm{T}$ its desired robustness against fluctuations of $V_{i n}$ which cause a comparator to oscillate when occurring close to its (single!) threshold voltage.

Clearly, the hysteresis behavior is tantamount to making the reference voltage depend on the current output state, which, in turn, implies that there is some kind of feedback of the output state to the input. Fig. 2 shows a typical CMOS implementation of a $\mathrm{S} / \mathrm{T}$ [4]. The feedback path via transistors $M_{3}$ and $M_{6}$ is clearly visible. It is exactly this (inevitable) existence of the positive feedback path that makes the S/T prone to metastability. In case of an input trace that first causes the $\mathrm{S} / \mathrm{T}$ state to flip but then, before the feedback path has fully stabilized, pulls the S/T back to the old state, undesired behavior can be observed at the output. As will be elaborated in more detail in Section IV, the S/T can exhibit the following behaviors at its output:

(B1) For strictly monotonic input traces the S/T will show clean (steep) and well timed output transitions. This is the regular behavior.

(B2) A monotonic input trace that brings the $\mathrm{S} / \mathrm{T}$ right to the tripping point and stays constant there can cause an indecision on whether to flip, which ultimately leads to a clean but (arbitrarily) late transition.

(B3) Similarly, an input trace that goes slightly beyond the threshold but then back again and stays constant, can make the $\mathrm{S} / \mathrm{T}$ assume a metastable state in which it outputs a constant voltage that is determined by the final constant value of $V_{i n}$ and may well be somewhere in between a clean HI and a clean LO.

(B4) An input pulse of marginal width, i.e. one that does not give the $\mathrm{S} / \mathrm{T}$ enough time to stabilize its state, can create a glitch at the output. Depending on whether this

\footnotetext{
${ }^{1}$ We will consider an inverting S/T throughout the paper, as virtually all practical implementations are inverting.
}

pulse reaches full height or not, we distinguish between a glitch and a runt, respectively.

(B5) In general, a non-monotonic input trace can, in principle, make the S/T output any desired voltage between clean $\mathrm{HI}$ and clean LO for any desired time - in fact by appropriately controlling the input trace one can obtain any desired output voltage trace (within the S/T's static and dynamic voltage limits, of course).

It very much depends on the application which of these behaviors are actually undesired, and as a consequence it is hard to quantify the improvement in signal quality obtained by the $\mathrm{S} / \mathrm{T}$ in general. In most cases marginal voltage levels are problematic, as they could be interpreted differently by different receivers. Unfortunately, in case (B3) one may still encounter marginal output voltage levels that are not just transient, but, as these cases are hard to trigger and hence can be considered rare, the $\mathrm{S} / \mathrm{T}$ provides a very good improvement in general. If output glitches are a concern, case (B4) becomes problematic, and even in case (B2) a late transition may form a glitch in conjunction with a regular subsequent one. Again, these cases need very specific input traces and are hence rare. Finally, due to case (B5), no type of output behavior can be completely ruled out by using the $\mathrm{S} / \mathrm{T}$, which means there is only a quantitative improvement, albeit a substantial one. However, as there are infinite numbers of both, "good" and "bad" traces, it is not possible to quantify the improvement; and we are not aware of any such approaches in the literature.

\section{QUESTIONS TO BE ANSWERED}

From the above analysis we have concluded that a single S/T stage improves the signal quality. So in principle, a subsequent $\mathrm{S} / \mathrm{T}$ stage should obtain a similar improvement, and thus overall the cascade has a higher gain than the single stage. However, it remains to be investigated whether this is indeed the case. In order to come to a conclusive answer, the following questions should be addressed:

(Q1) In which cases does the second S/T stage improve the behavior?

(Q2) Are there cases in which the behavior is getting worse? Are there new types of (likely) behavior?

(Q3) Is the second stage equally likely to become metastable as the first one?

(Q4) Can metastability of the last stage be completely avoided, possibly by forming a longer cascade?

(Q5) How are the static properties of the cascade determined (is it still a $\mathrm{S} / \mathrm{T}$, and if so, which hysteresis)?

(Q6) How are the dynamic properties determined (regular delay, output slope, is there a performance penalty in using a cascade?)

(Q7) Are there any rules for optimal dimensioning of the cascade (combination of fast and slow stage, different hystereses,...)

To conclusively answer these questions we will in the following elaborate a sufficiently detailed understanding of the behavior of a (2-stage) S/T cascade. As a first step towards 


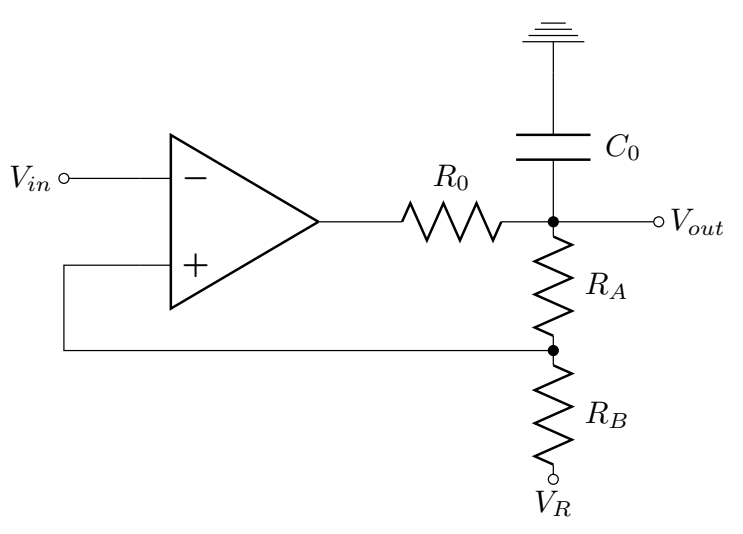

Fig. 3. Dynamic model of the S/T inspired by Marino [5]

this end we will revisit an existing model for the metastable behavior of a single $\mathrm{S} / \mathrm{T}$ stage in the next section.

\section{Metastability model of a Schmitt-Trigger STAGE}

Marino has already proposed a dynamic model for the S/T that allows to investigate its metastable behavior [5]. This model is based on a $\mathrm{S} / \mathrm{T}$ implementation using an operational amplifier (OpAmp) as shown in Fig. 3. The OpAmp is assumed to be ideal, but with a limited output voltage range of $\pm M$. An RC low pass at its output defines its dynamic behavior, and a resistive voltage divider by $k \in\{0 \ldots 1\}$ feeds back part of the output voltage to the input (positive feedback). For brevity we omit the derivation and solution of the associated differential equations here (for details please refer to [5]) and just show the results:

As the saturation requires separate treatment, the solution comprises three regions, as illustrated in Fig. 4: upper and lower saturation (Regions 1 and 3), as well as the "linear region" 2 between them. The dashed lines represent the borders between the regions (the corresponding equations are also given).

The dynamic system represented by the $\mathrm{S} / \mathrm{T}$ is then described by the following equations:

$$
\text { Region 1: } \quad \frac{d V_{\text {out }}}{d t}=V_{\text {out }}^{\prime}=-\frac{1}{\tau_{1}}\left(V_{\text {out }}-\gamma_{1}\right)
$$

The resulting trajectory for $V_{\text {out }}$ is a decaying exponential function with time constant $\tau_{1} \approx R_{0} C_{0}$ that asymptotically approaches the truly stable rest point $\gamma_{1} \approx M$.

$$
\text { Region 2: } \quad \frac{d V_{\text {out }}}{d t}=V_{\text {out }}^{\prime}=\frac{1}{\tau_{2}}\left(V_{\text {out }}-\gamma_{2}\right)
$$

Here we have a growing exponential function with time constant $\tau_{2} \approx \frac{R_{0} C_{0}}{k A-1}$ that moves away from the metastable rest point $\gamma_{2} \approx \frac{V_{i n}-(1-k) V_{R}}{k-\frac{1}{A}}$. Note that we do not have a single metastable point, as in case of a latch, but all points on $\gamma_{2}$ are metastable points and the actual rest point depends on $V_{\text {in }}$ (see Fig. 4).

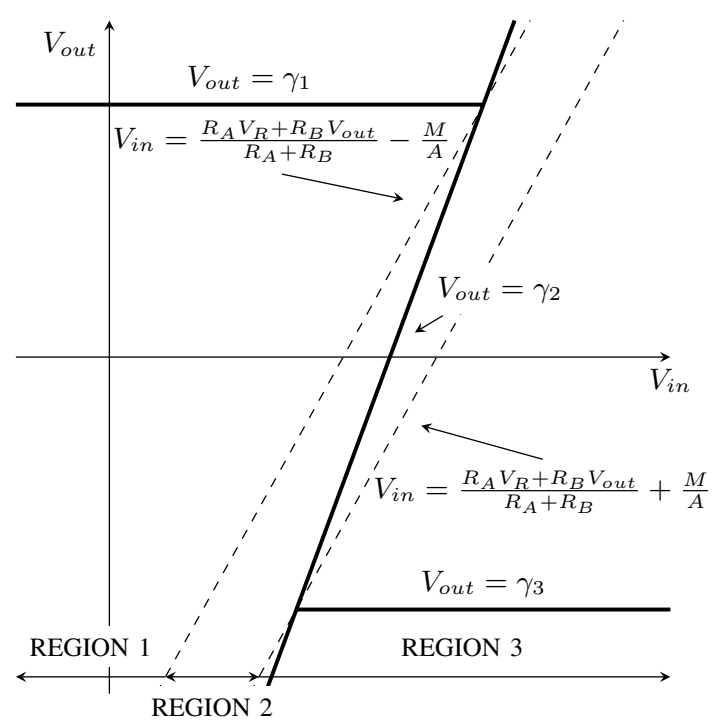

Fig. 4. Phase diagram for the S/T from Marino [5]

$$
\text { Region 3: } \quad \frac{d V_{\text {out }}}{d t}=V_{\text {out }}^{\prime}=-\frac{1}{\tau_{3}}\left(V_{\text {out }}-\gamma_{3}\right)
$$

Similar to Region 1 this yields a decaying exponential function with time constant $\tau_{3}=\tau_{1} \approx R_{0} C_{0}$ that asymptotically approaches the truly stable rest point $\gamma_{3}=-\gamma_{1} \approx-M$.

At this point it is interesting to compare the $\mathrm{S} / \mathrm{T}$ with a latch, as Veendrick derived a similar model for latches in [7] to investigate their metastability behavior. While both elements have a positive feedback, the key difference is that in the latch the input becomes decoupled when the storage loop is closed (i.e. when switching to hold mode). Mathematically this means that the input becomes irrelevant, and just the homogeneous solution of the differential equation applies. In the S/T this is not the case, and the input continuously influences the behavior. This not only complicates the mathematical treatment (and is probably the key reason why there are no quantitative improvement values available for the $S / T$ ), it also results in the S/T having more than one metastable point: depending on the input voltage the $S / T$ can rest in any metastable location along the $\gamma_{2}$ line and hence produce any arbitrary metastable output voltage. Finally, this lack of decoupling between stages also complicates the analysis of the $\mathrm{S} / \mathrm{T}$ cascade.

As a physical analogy one might consider the metastable latch as a stick that is vertically placed on a firm table such that it balances on its tip before it finally falls, while the S/T is the same vertical stick but balancing on a finger that can still be moved left and right (inverted pendulum).

\section{Claims about the Behavior of A SCHMITT-TRIGGER CASCADE}

In the following we want to investigate a two stage S/T cascade as shown in Fig. 5. It was already argued in Section II that a single $\mathrm{S} / \mathrm{T}$ stage can essentially exhibit any output behavior (case (B5)). This means that the first stage does not 


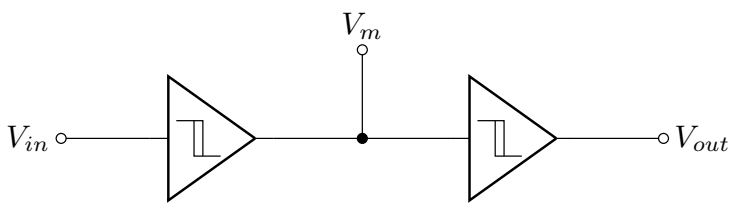

Fig. 5. Two stage S/T cascade

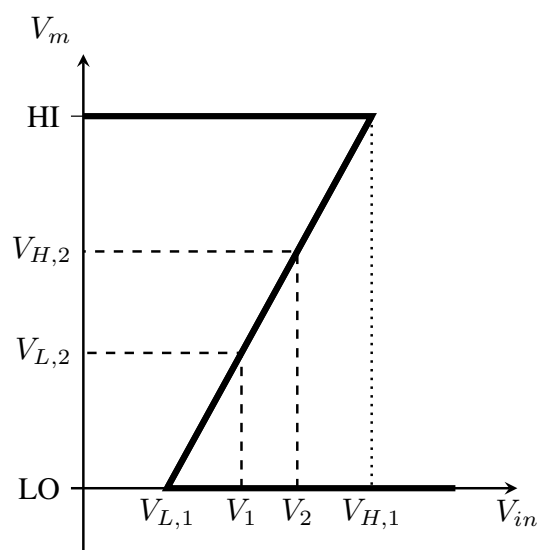

Fig. 6. Characteristic of a single $\mathrm{S} / \mathrm{T}$ showing $V_{1}$ and $V_{2}$

qualitatively restrict the second one's input space, and, as a consequence, stage 2 has unrestricted output behavior as well. So at this point we can already answer question (Q4) about complete avoidance of metastability through a S/T cascade: This is simply not possible.

In continuation of the physical analogy given in Section IV we can view the second stage as a second vertical stick balancing on the upper tip of the first one. This analogy nicely illustrates that it becomes much more unlikely to see metastability in the second stage (i.e. actually find a balance for the second stick) - thus giving an intuitive answer to (Q3) - , but it is physically possible.

To get closer to a quantitative answer, let us analyze how the different output behaviors of the first stage are handled by the second one. The internal signal connecting the $\mathrm{S} / \mathrm{T}$ is named $V_{m}$ ( $c f$. Fig. 5).

\section{A. Regular behavior}

Let us start with the regular behavior (B1) and assume a starting point with $V_{i n}<V_{L, 1}$ (the case of $V_{i n}>V_{H, 1}$ is analogous). Note that in $V_{L, i}$ and $V_{H, i}$ the index $i$ corresponds to the stage number. Due to the inverting behavior of each of our S/T stages, we have $V_{m}$ at $\mathrm{HI}$ and $V_{\text {out }}$ at LO again. As $V_{\text {in }}$ increases, $V_{m}$ and $V_{\text {out }}$ stay constant until $V_{\text {in }}$ reaches $V_{H, 1}$. Beyond that point $V_{m}$ will switch to LO. With a strictly monotonic $V_{i n}$ this transition of $V_{m}$ will be rapid. Clearly, this transition of $V_{m}$ will also cause the second stage to switch, namely when crossing its threshold $V_{L, 2}$. Overall, we experience a clean switching, with the threshold determined by that of the first stage, while the second stage's threshold is irrelevant, as $V_{m}$ crosses the whole voltage range anyway. This answers question (Q5).

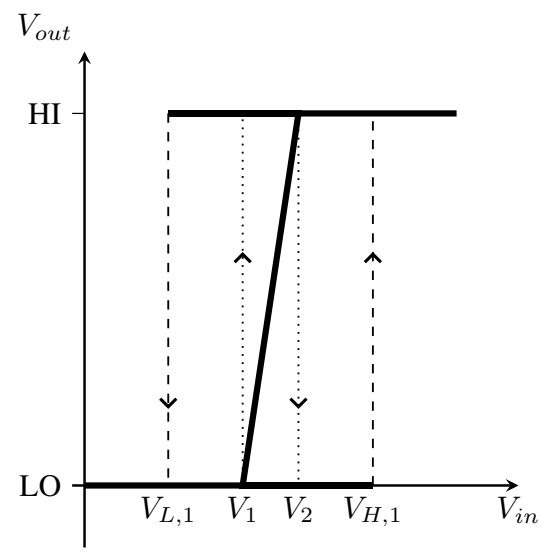

Fig. 7. Theoretical hysteresis $\left(V_{i n}-V_{\text {out }}\right)$ of $\mathrm{S} / \mathrm{T}$ cascade

Generally, in this mode of operation we can expect the steepness of the transitions to increase as the first $\mathrm{S} / \mathrm{T}$ tends to switch fast when its threshold is reached, causing the second one to change even faster. The signal is however delayed by the propagation delay of the second $\mathrm{S} / \mathrm{T}$.

\section{B. Late transitions of stage 1}

According to case (B2) a ramp input stopping at a constant value near the threshold will cause a late but clean transition at stage 1 . In that case the second stage perceives a clean input which it simply conveys (adding its nominal propagation delay). So late transitions are essentially not modified by the second stage.

\section{Hysteresis curve of the cascade}

We know from Section II that for a single S/T to become metastable its input voltage must be between its thresholds. This means we can only make the second stage metastable with a $V_{m}$ between $V_{L, 2}$ and $V_{H, 2}$, which will (apart from a steep transition during switching as described above) only occur when the first stage is metastable, and even then only in a specific range, as shown in Fig. 6. In the following we will use $V_{1}$ and $V_{2}$ to denote those values of $V_{i n}$ that, if the first $\mathrm{S} / \mathrm{T}$ is metastable, will cause it to output $V_{m}=V_{L, 2}$ and $V_{H, 2}$ respectively. This consideration allows us to draw the hysteresis curve for the overall behavior of the cascade shown in Fig. 7: Still the static switching points are determined by the first stage, but the range of possible metastable behavior is limited to the range of $V_{1} \leq V_{\text {in }} \leq V_{2}$, with $V_{1}$ and $V_{2}$ being determined by the slope of the $\gamma_{2}$ line of the first stage and the thresholds of the second stage.

The same hysteresis is achieved when using two noninverting Schmitt-Triggers, while combining an inverting S/T with a non inverting one, mirrors the hysteresis around the line $V_{\text {out }}=(H I+L O) / 2$, independent of the ordering (assuming equal $V_{L, i}$ and $V_{H, i}$ values).

For the case that the hystereses of the single S/Ts are not equal the ordering is important, as $V_{H}$ and $V_{L}$ of the cascaded system are determined by the first $\mathrm{S} / \mathrm{T}$ alone and $V_{1}$ and $V_{2}$ by 
both of them. Let $V_{N i}$ be $\left(V_{H, i}+V_{L, i}\right) / 2$. If $V_{N 1}=V_{N 2}=$ $V D D / 2$ the order does not have an influence on $V_{1}$ and $V_{2}$. In all other cases they might deviate, however the difference between them, i.e. $V_{2}-V_{1}$, is constant. This is very important since $V_{2}-V_{1}<V_{H}-V_{L}$. Therefore it is possible, if the first $\mathrm{S} / \mathrm{T}$ is held in metastability, to create a pulse train at the output with the reduced hysteresis $V_{1}$ to $V_{2}$ (cycling the dotted lines in Fig. 7).

\section{Moving into metastability}

To move the second stage into metastability, stage 1 needs to be made metastable first. This can be attained by increasing $V_{i n}$ from a value lower than $V_{L, 1}$ to $V_{H, 1}$ and then decreasing it appropriately, while stage 1 starts to switch (behavior (B3)). By staying close to the metastable restpoints $\left(\gamma_{2}\right.$ in Fig. 4) until $V_{m}$ reaches $V_{L, 2}\left(V_{i n}=V_{1}\right)$ the second S/T will start to switch and can be driven into metastability in the same way as the first one. While keeping both S/Ts in metastability, which is either possible with very precise or very fast control of $V_{i n}$ (for a more detailed explanation see [6]), the output values between LO and HI shown in Fig. 7 are reachable.

\section{E. Emergence of glitches}

An interesting behavior can be observed when, with both stages in the metastable state, $V_{i n}$ is increased to a value between $V_{2}$ and $V_{H}$. This will bring $V_{m}$ to above $V_{H}$ and hence cause the second stage to flip to LO. A further (monotonic!) increase of $V_{i n}$ beyond $V_{H}$ will then bring the first stage to saturation and make $V_{m}$ transition to LO, which, in turn, causes the second stage to flip back to HI. In this case we have observed a glitch at $V_{\text {out }}$ that was caused by a monotonic transition of $V_{i n}$ (however, a non-monotonic $V_{i n}$ was initially required to bring both $\mathrm{S} / \mathrm{T}$ s into the metastable state in the first place). The root of this behavior lies in the positive slope of the $\gamma_{2}$ line, which is somehow contradictory to the otherwise inverting behavior of the $\mathrm{S} / \mathrm{T}$. This is also expressed by the direction of the arrows in Fig. 7.

While this definitely represents a new type of (often undesired) behavior not seen with a single stage - thus answering (Q2) positively - one should keep in mind that it takes an extremely precise control of $V_{i n}$ to navigate into this case. In the physical analogy this would equal the case of bringing both sticks in vertical balance and then having them fall down to opposite sides.

\section{F. Generalization of the analysis}

The above scenarios only represent some selected possible cases of output behavior. More generally, the shape of $V_{\text {out }}(t)$ is determined by (a) the shape of the input voltage $V_{i n}(t)$, (b) the state of stage 1 (given by $V_{m}(t)$ ), and (c) the state of stage 2 (given by $V_{\text {out }}(t)$ ). In this 3-dimensional space one can identify 9 characteristic sub-spaces (for $V_{i n}$ remaining constant at its initial value). Five of these are listed in Table I, the other 4 are symmetric and not treated here for brevity. The subtables (1)...(5) apply for different values of $V_{i n}$. Table (1) shows the case where $V_{i n}$ is significantly lower than $V_{L, 1}$, such that $V_{m}$ is forced to $\mathrm{HI}$ and consequently $V_{\text {out }}$ to $\mathrm{LO}$ immediately, irrespective of their initial state. The zeros denote the final state of the cascade's output. The arrows show what event could be observed at the output. 0 means the output was initialized to zero and remains there, $0 \downarrow$ means the output makes a fast transition to 0 from wherever it was initialized to. The $0 \_$shows that there is (very limited) glitch potential if the nominal propagation delays of the two S/Ts significantly vary.

TABLE I

POSSIBLE OUTPUT BEHAVIORS WITH STATIC INPUT

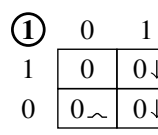

\begin{tabular}{l|c|c|}
2 & \multicolumn{1}{c}{0} & 1 \\
\cline { 2 - 3 } 1 & 0 & $0 \downarrow$ \\
\cline { 2 - 3 } 0 & $0 \Omega$ & $0 \downarrow$ \\
\hline
\end{tabular}
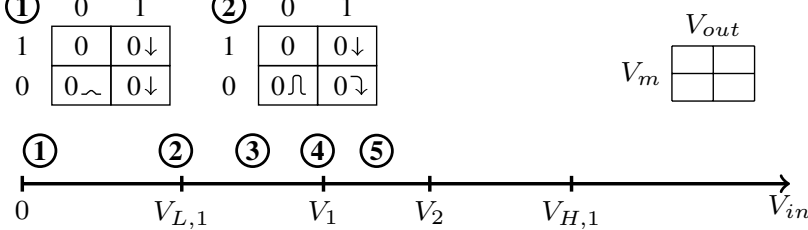

\begin{tabular}{|c|c|c|}
\hline (3) & 0 & 1 \\
\hline 1 & 0 & $0 \downarrow$ \\
\hline$r$ & $0 \Omega$ & 02 \\
\hline$\rightarrow$ & $1 \uparrow$ & 1 \\
\hline ב & $1 \uparrow$ & 1 \\
\hline 0 & $1 \uparrow$ & 1 \\
\hline
\end{tabular}

\begin{tabular}{l|c|c|} 
(4) & \multicolumn{1}{c}{0} & \multicolumn{1}{c}{1} \\
\cline { 2 - 3 } 1 & 0 & $0 \downarrow$ \\
\cline { 2 - 3 }$\neg$ & $0 \widetilde{\Omega}$ & $0 \downarrow$ \\
\cline { 2 - 3 }$\rightarrow$ & $1 \uparrow$ & 1 \\
\cline { 2 - 3 }$\curvearrowright$ & $1 \uparrow$ & 1 \\
\cline { 2 - 3 } 0 & $1 \uparrow$ & 1 \\
\cline { 2 - 3 } & &
\end{tabular}

\begin{tabular}{|c|c|c|c|c|c|}
\hline (5) & 0 & \multirow[t]{2}{*}{ 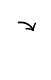 } & \multirow[t]{2}{*}{$\rightarrow$} & \multirow[t]{2}{*}{$\pi$} & 1 \\
\hline 1 & 0 & & & & $0 \downarrow$ \\
\hline & 0 & \૪ & $0 \triangleleft$ & $0 \precsim$ & $0 \beth$ \\
\hline & 0 & $0_{\searrow}$ & $\rightarrow$ & $1^{r}$ & 1 \\
\hline & $1 \uparrow$ & $1 \approx \lambda$ & $1^{\nearrow}$ & $1^{\top}$ & 1 \\
\hline & $1 \uparrow$ & & & & 1 \\
\hline
\end{tabular}

Table (2) shows possible outputs when $V_{i n}$ is so close to $V_{L, 1}$ that it causes an abnormal switching delay of the first S/T (Region 2 in Fig. 4). Due to this delay, a late transition $(0 \neg)$ or a glitch $(0 \Omega)$ can be observed at the output when $V_{m}$ is initialized to 0 . That would correspond to the case of glitch emergence described above.

In table (3), $V_{i n}$ is between $V_{L, 1}$ and $V_{1}$ - the first stage can now also be initialized metastable resolving to $1(\neg)$, to $0(\curvearrowright)$ or not resolving at all $(\rightarrow)$. As can be seen, due to $V_{\text {in }}<V_{L, 1}$ the metastable voltage must be below $V_{L, 2}$ for the next stage, which can therefore not distinguish this type of metastability from a clean LO. When metastability resolves to HI, the effect on the second stage will be identical to the late transitions in the previous paragraph.

Table (4) shows the case when the metastable voltage output of the first stage is very close to $V_{L, 2}$. The difference to the previous table is that during metastability of the first $\mathrm{S} / \mathrm{T}$, the second one can delay its output transition. The most interesting case is $0 \bar{\Omega}$. It shows that the relationship of the delays (metastability/late transition) makes a qualitative difference at the output: If $V_{m}$ reaches $\mathrm{HI}$ first, the output remains LO; if $V_{\text {out }}$ is faster, the output could be an arbitrarily delayed glitch.

Table (5) presents the behaviors for input voltages of $V_{1}<$ $V_{\text {in }}<V_{2}$. Here both $\mathrm{S} / \mathrm{Ts}$ can be initialized metastable, therefore the table has many entries. Apart from late transitions, $0 \approx$ and $1 \approx$ again show the interesting cases of race conditions. Depending on the order of metastability resolution, the output can either go directly from an undefined voltage to $\mathrm{HI}$ or LO (first S/T resolves before second one), or first go to the opposite logic state followed by a full range transition (second $\mathrm{S} / \mathrm{T}$ resolves before first one), thus generating a glitch. 

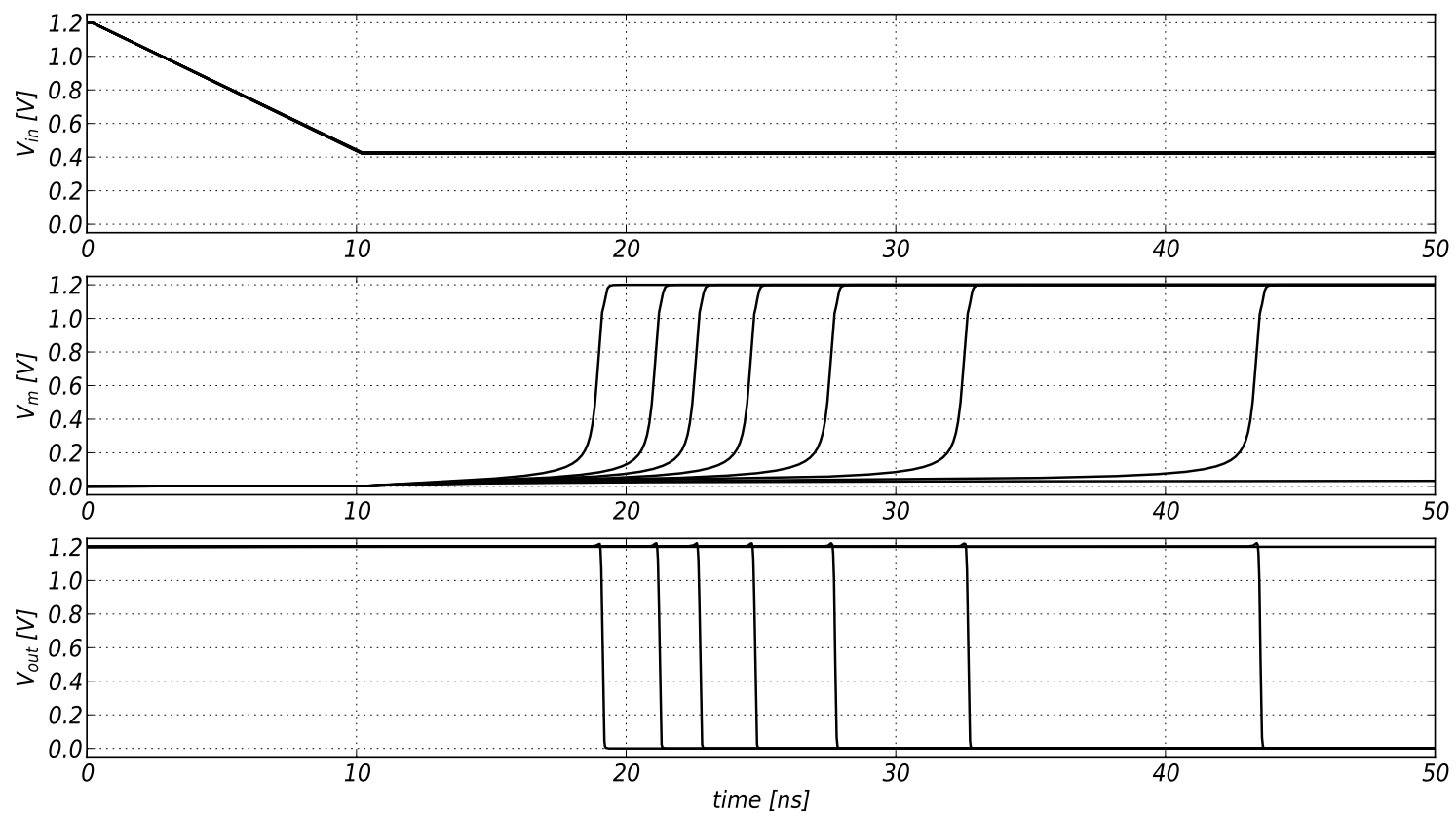

Fig. 8. Time trace of $V_{i n}, V_{m}$ and $V_{\text {out }}$ for input slopes stopping at a constant value near $V_{L, 1}$

So we can conclude that in addition to the 3-dimensional space described so far, the order of metastability resolution is important as well, if both $\mathrm{S} / \mathrm{Ts}$ are metastable.

As a general trend we can observe in the table that, while some of the metastable cases of stage 1 are propagated, others are turned into other behaviors like proper transitions, delayed transitions, or glitches. In no case an intermediate voltage is generated from clean transitions. So in applications where the key purpose of using a $\mathrm{S} / \mathrm{T}$ lies in protecting the subsequent logic from intermediate voltages, the cascade does a decent job in reducing that risk. However, if glitches and badly timed transitions are dangerous, then the use of the cascade may be counter-productive. This may be considered an answer to (Q1) ... (Q3).

\section{G. Pulse propagation}

According to case (B4) a single S/T stage may or may not propagate a glitch or runt. As the second stage may, of course, show the same behavior, glitches and runts may propagate through the whole cascade. As the second stage may turn some of these into stable transitions, however, the probability of propagation can be expected to become lower in the cascade.

\section{VALIDATION OF THE CLAIMS}

To validate our predictions about the behavior of the cascade from the previous section, we performed HSPICE simulations of two S/Ts in series, each implemented using the circuit shown in Figure 2 with transistor parameters of an industrial $65 \mathrm{~nm}$ process.
Fig. 8 shows the behavior of the cascaded S/Ts when a ramp resulting in a constant value is applied to the input (case (B2)). As one can see the first stage responds with late but clean transitions, i.e. ones that cross the intermediate voltage range sufficiently fast. The second stage then increases the steepness of the transitions even further. Please note that the inputs causing the different traces only deviate by a very small amount of their final, constant value. Therefore we can confirm, that $V_{H, 1}$ respectively $V_{L, 1}$ has to be approached very accurately to observe late transitions at the output. However, this is due to the properties of the first stage alone, while the second stage does not yield any further improvement.

When short pulses are applied to the cascaded S/Ts (case (B4)), these are preserved or suppressed depending on their width, as can be seen in Fig. 9. A little bit misleading is the fact, that the pulses at the output seem to be longer than those at the input, since the time they spend below $V_{L}$ is longer than for those at the input. This can, however, be explained by the increased steepness of the transitions. When the pulses are compared by their crossing times of $V D D / 2$, for example, one can see that they stayed the same and are not stretched in time.

Figures 10 and 11 show the cases when both $\mathrm{S} / \mathrm{Ts}$ are in their metastable region and the first respectively second one resolves before the other. In the first case $V_{\text {out }}$ increases with $V_{m}$ until it reaches $V_{H}$, where $V_{\text {out }}$ drops rapidly. If, however, the second $\mathrm{S} / \mathrm{T}$ resolves first and afterwards the first one to the same value, a glitch at the output is introduced. This exactly matches our predictions from theory. 

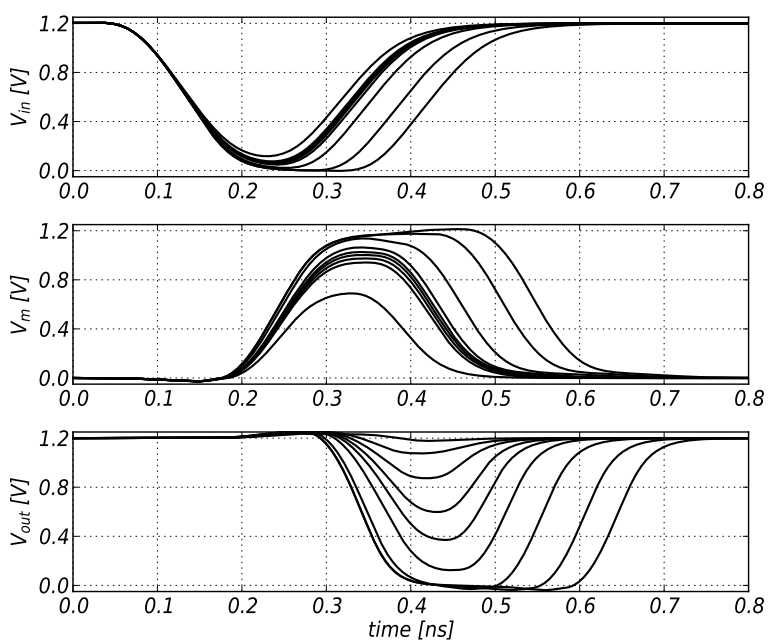

Fig. 9. Time trace of $V_{i n}, V_{m}$ and $V_{\text {out }}$ for pulse inputs

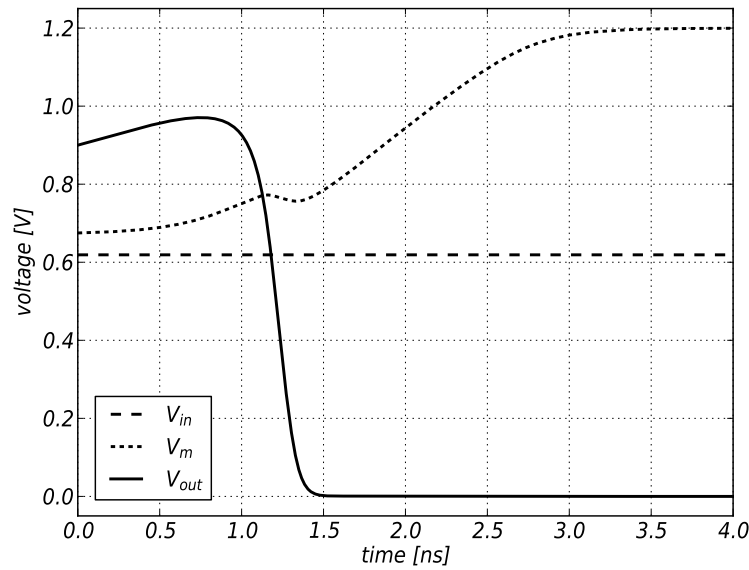

Fig. 10. First stage resolving earlier from metastability

In the simulation we also observed a behavior that was not predicted, namely that transitions of stage 2 influence the behavior of stage one. This coupling can be seen best in Fig. 12. Initially the first Schmitt-Trigger resolves towards VDD until $V_{H, 2}$ is reached, causing the second one to switch. This, however, also introduces a change at the output of stage one forcing it to drop.

Fig. 13 shows how the $\mathrm{S} / \mathrm{T}$ cascade can be driven into metastability and even forced to output arbitrary waveforms (in this example a sine wave). The beginning of the figure (first $15 \mathrm{~ns}$ ) shows regular transitions (case (B1)). The hysteresis is clearly observable and it also becomes clear why the cascade's thresholds/hysteresis are determined by those of the first S/T in the series - it applies its hysteresis to the input and outputs fast, full range transitions. With such transitions at its input, the second S/T's hysteresis only marginally increases the propagation delay through the cascade but does not alter the

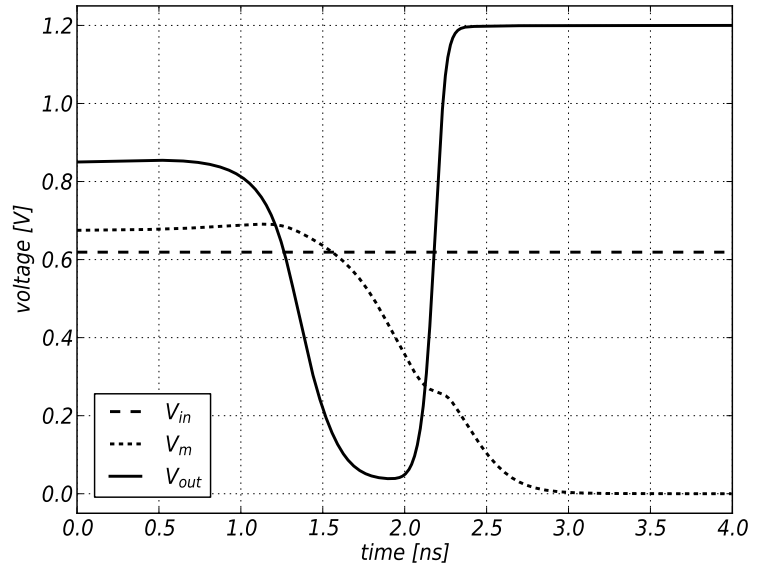

Fig. 11. Second stage resolving earlier from metastability

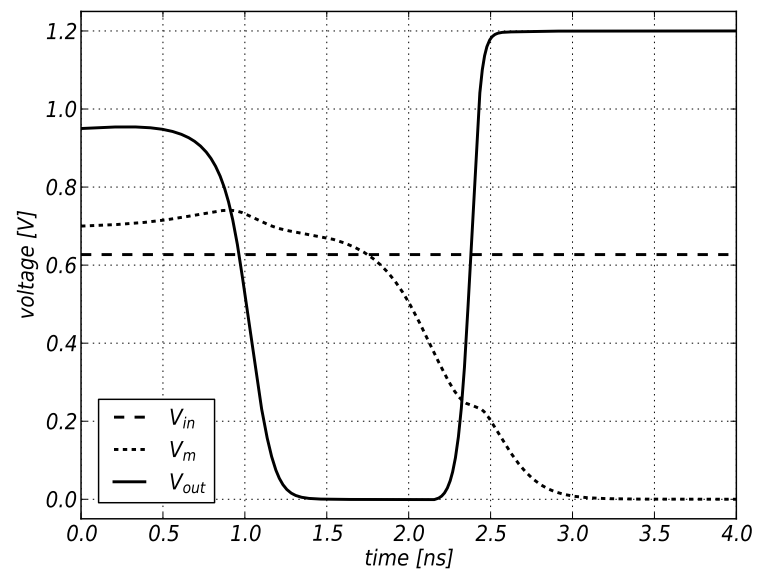

Fig. 12. Interactions between switching $\mathrm{S} / \mathrm{T}$ stages

overall hysteresis.

The next part of the figure (until $28 \mathrm{~ns}$ ) shows how the first $\mathrm{S} / \mathrm{T}$ is driven into metastability by carefully reverting $V_{i n}$ when $V_{m}$ begins to switch. The waveform that the metastable $\mathrm{S} / \mathrm{T}$ outputs is such that the second $\mathrm{S} / \mathrm{T}$ becomes metastable in the same manner (from $28 \mathrm{~ns}$ onward). With both $\mathrm{S} / \mathrm{Ts}$ metastable, the cascade is driven to output a sine wave (case (B5)) followed by a constant output voltage (case (B3)). During the sine output the non-inverting behavior of the single S/T stages and the amplification of $V_{\text {in }} t o V_{m}$ and then $V_{\text {out }}$ can be observed very clearly. In the end (from $70 \mathrm{~ns}$ ) the second S/T is resolving to VDD after which the first $\mathrm{S} / \mathrm{T}$ also resolves to VDD, forcing $V_{\text {out }}$ to GND. The input is continuously increased and eventually crosses $V_{H, 1}$, causing one last output transition - again as predicted in theory.

Finally, Fig. 14 depicts the measured stable (truly stable plus metastable) points in the $\left(V_{\text {in }}, V_{m}, V_{\text {out }}\right)$-space. The gray line at the back is the projection of the 3D-curve to the plane $V_{m}$ over $V_{i n}$ and thus represents the hysteresis curve of the 


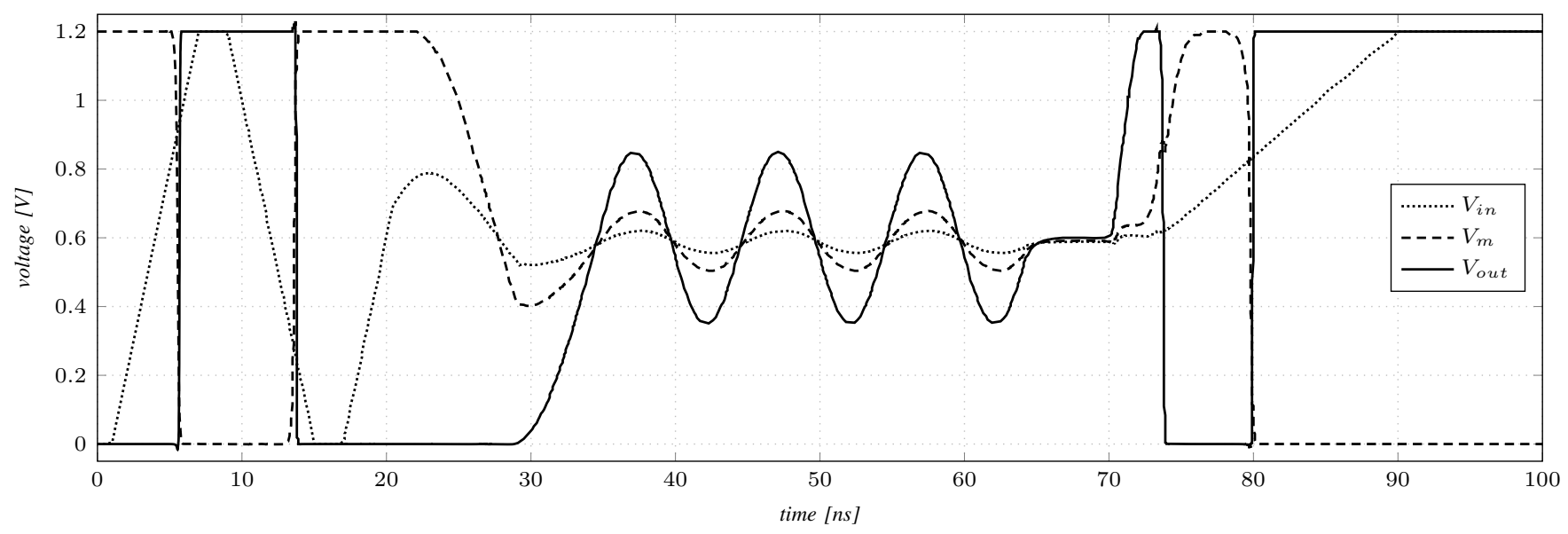

Fig. 13. "Arbitrary" waveform created by operating both S/Ts in metastability

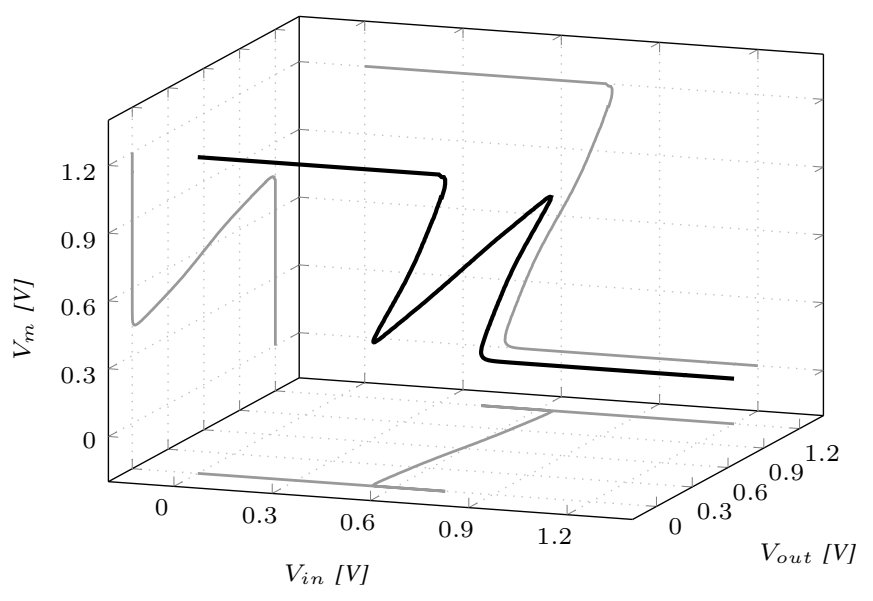

Fig. 14. Characteristic of the cascade

first stage. The projection to the left plane $\left(V_{m}\right.$ over $\left.V_{\text {out }}\right)$ shows (a rotated version of) the hysteresis curve of the second stage. The most interesting one is the projection of the curve to the ground plane, i.e. $V_{\text {out }}$ over $V_{\text {in }}$. This represents the overall hysteresis of the cascade. And indeed this curve exactly matches our prediction from Fig. 7.

The 3D view also gives a better understanding of the reachability of the metastable states with $V_{\text {out }}$ neither HI nor LO. These are only the states on the line connecting $\left(V_{\text {in }}, V_{m}, V_{\text {out }}\right)=(0.64,0.74,1.2) \mathrm{V}$ and $(0.53,0.43,0) \mathrm{V}$. This line segment has two noteworthy properties: 1) Both its projections to the first and second $\mathrm{S} / \mathrm{T}$ characteristic coincide with metastable states - the output can only be held at an intermediate voltage when both $\mathrm{S} / \mathrm{Ts}$ are metastable. 2) It does not start from any stable state. This is not apparent in the $2 \mathrm{D}$ overall cascade characteristic. To reach any point from that segment in a controlled manner, the first $\mathrm{S} / \mathrm{T}$ has to be metastable for a considerable time to produce the non-monotonic waveform required to make the second S/T metastable. Intuitively, both arguments lead to a substantially lower probability of the $\mathrm{S} / \mathrm{T}$ cascade to output an intermediate voltage compared to a single $\mathrm{S} / \mathrm{T}$.

\section{CONCLUSION}

We have addressed the question whether the cascading of Schmitt-Trigger stages improves the metastable behavior. Our comprehensive theoretical and simulation-based analysis showed that the risk of an intermediate output voltage is indeed decreased, and generally the probability of metastable behavior is significantly reduced. This is due to the fact that the first stage must necessarily be metastable for the second stage to become metastable as well. However, there are cases in which the interaction of the two states of the two S/T stages causes extra transitions, and also some cases of intermediate voltage are converted to transitions by the second stage. If the resulting, potentially misplaced transitions are a problem, the usefulness of the cascade should be carefully reconsidered in the given application context.

Another result is that metastable output behavior cannot be safely ruled out, even with an arbitrarily long cascade. We have shown for the two-stage cascade that an arbitrary output waveform can be generated, if only the input is controlled precisely enough.

\section{REFERENCES}

[1] L. R. Marino, "General Theory of Metastable Operation," IEEE Transactions on Computers, vol. C-30, no. 2, pp. 107-115, Feb. 1981.

[2] L. Kleeman and A. Cantoni, "Metastable Behavior in Digital Systems," IEEE Design Test of Computers, vol. 4, no. 6, pp. 4-19, Dec 1987.

[3] R. Ginosar, "Metastability and synchronizers: A tutorial," IEEE Design Test of Computers, vol. 28, no. 5, pp. 23-35, Sept 2011.

[4] I. Filanovsky and H. Baltes, "CMOS Schmitt trigger design," IEEE Transactions on Circuits and Systems I: Fundamental Theory and Applications, vol. 41, no. 1, pp. 46-49, Jan 1994.

[5] L. R. Marino, "The Effect of Asynchronous Inputs on Sequential Network Reliability," IEEE Transactions on Computers, vol. 26, no. 11, pp. 10821090, 1977.

[6] A. Steininger, J. Maier, and R. Najvirt, "The metastable behavior of a schmitt-trigger," in 22nd IEEE International Symposium on Asynchronous Circuits and Systems (ASYNC), May 2016, pp. 57-64.

[7] H. J. Veendrick, "The behaviour of flip-flops used as synchronizers and prediction of their failure rate," IEEE Journal of Solid-State Circuits, vol. 15, no. 2, pp. 169-176, Apr 1980. 